\section{Pyoderma Gangrenosum and lymph nodes tuberculosis disease: unusual association}

\author{
Inès Zaraa, ${ }^{1,2}$ Abdulmohti Hawilo, ${ }^{1}$ \\ Slim Ben Hassine, ${ }^{1}$ Inès Chelly, ${ }^{2,3}$ \\ Slim Haouet, 2,3 Mokni Mourad, ${ }^{1,2}$ \\ Amel Ben Osman 1,2 \\ 'Dermatology department, La Rabta \\ Hospital, Tunis; 'Faculté de medicine de \\ Tunis, Université El Manar, Tunis; \\ ${ }^{3}$ Pathology department La Rabta \\ Hospital, Tunis, Tunisia
}

\begin{abstract}
Pyoderma gangrenosum (PG) is a rare neutrophilic dermatosis with unknown etiology. PG associated with infectious disease is very unsual. We report a singular case of PG with lymph nodes tuberculosis disease.
\end{abstract}

\section{Case Report}

A previously healthy 60 -year-old man presented with a 3-month history of numerous ulcers in the limbs. No history of trauma or disorders of digestive transit or tuberculosis was noted.

Clinical exam revealed multiple ulcers on the right lower leg, with necrotic demarcation and undermined borders surrounded by a lividerythematous coloured margin (Figure 1A).

Repeated sputum cultures for bacterial, fungi, and mycobacterial organisms yielded no growth. A skin biopsy showed histological images consistent with PG (Figure 1B,C). Chest radiography was normal. Chest and abdominal computerized tomographic (CT) scan revealed multiple supraclavicular, mediastinal and abdominal lymph nodes with central necrosis (Figure 2A). Tuberculin skin testing was positive. A supraclacicular lymph node biopsy showed lymph nodes tuberculosis (Figure 2B).

Routine laboratory investigations revealed neutrophilia (13000 elements/ $/ \mathrm{mm}^{3}$ ). The erythrocyte sedimentation rate was $72 \mathrm{~mm} / \mathrm{h}$. Liver, kidney, thyroid function tests, immunoglobulin, protein electrophoresis, anticoagulation panel, and tumor markers were normal. Venereal Disease Research Laboratory tests, HIV test, anti-neutrophilic cytoplasmic, antinuclear and anti-DNA antibodies, rheumatoid factor, cryoglobulins and serologic screening tests for viruses, Mycoplasma, Legionella, Chlamydia, Aspergillus, and Candida were all negative.
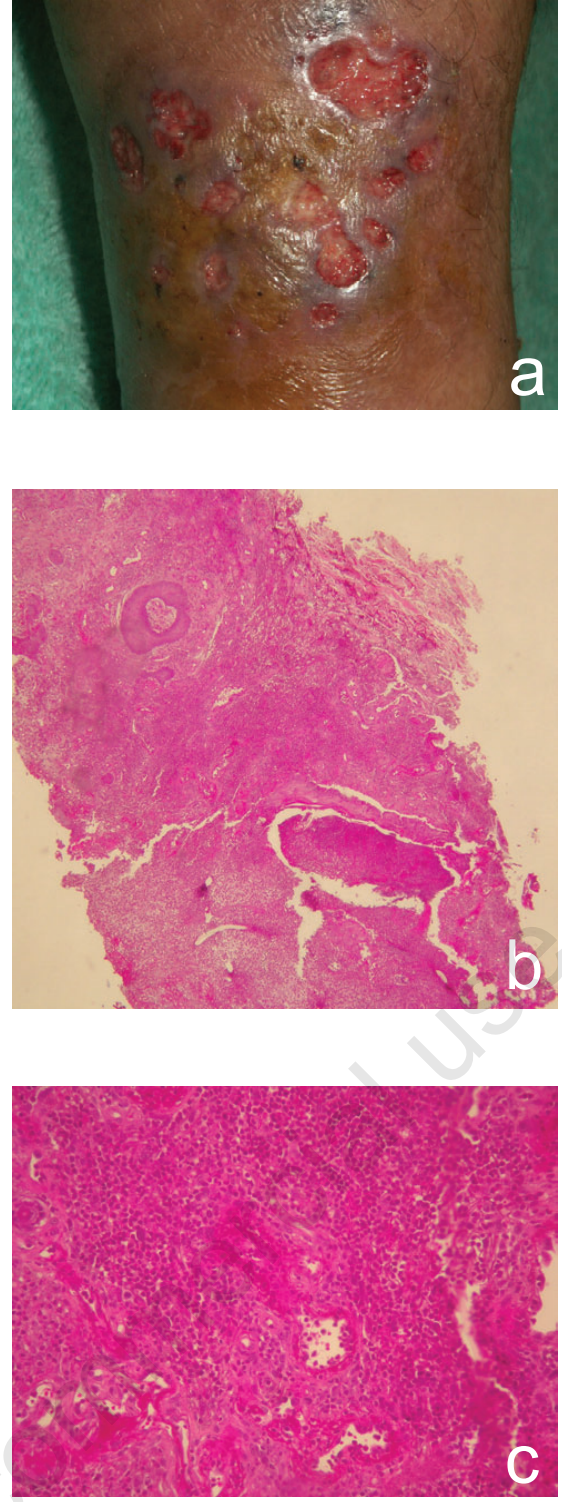

Figure 1. (A) Numerous ulcers on the right lower leg with a fibropurulent base and violaceous, partially necrotic border. (B) Skin biopsy specimens reveal oedema and massive neutrophilic inflammation with necrosis, haemorrhage and engorgement of vessels (HEx 40). (C)The extremely dense infiltrate of polymorphonuclearleucocytes leads to abscess formation and liquefaction necrosis of the tissue (HEx100).

There was no evidence for involvement of the respiratory tract or for renal disease as a possible manifestation of Wegener's granulomatosis or tuberculosis. In addition to the local wound management, the patient was treated with antituberculous drugs including isoniazid $300 \mathrm{mg}$ daily, rifampicin $600 \mathrm{mg}$ daily, pyrazinamide $2 \mathrm{~g}$ daily and ethambutol $800 \mathrm{mg}$ daily. During a 5-month follow-up, the skin ulcerations improved with re-epithelialization.

According to the clinical appearance, the rate of progression, and the histopathological
Correspondence: Inès Zaraa, Dermatology Department, La Rabta, Hospital, Jabbari, Bab Saadoun, Tunis, 1007 Tunisia.

Tel. +216.98307425 - Fax. +21671569449 .

E-mail: inesrania@myway.com

Key words: Pyoderma Gangrenosum, lymph nodes tuberculosis.

Received for publication: 18 May 2011.

Revision received: 10 June 2011.

Accepted for publication: 15 June 2011.

This work is licensed under a Creative Commons Attribution NonCommercial 3.0 License (CC BYNC 3.0).

(C) Copyright I. Zaraa et al., 2011

Licensee PAGEPress, Italy

Dermatology Reports 2011; 3:e8

doi:10.4081/dr.2011.e8
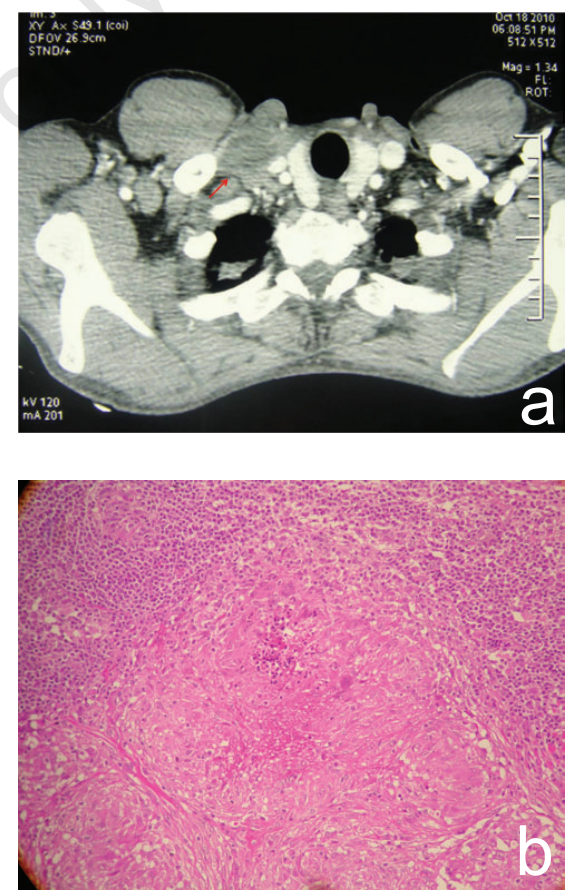

Figure 2. (A) Chest (CT) scan: large right supraclavicular lymph node with peripheral enhancement (red arrow) and central low-density. (B) Haematoxylin and eosin stained sections of lymph nodes revealed distinct and well-formed epithelioid cell granulomasGiant cells with central caseation necrosis (HEx100).

findings, our patient an ulcerative form of PG associated with lymph nodes tuberculosis disease. Over fifty percent of patients with PG have an underlying systemic disease, most commonly inflammatory bowel disease, hematological malignancies, or autoimmune disorders, which were excluded in our case.

Infection such as viral infection (HIV, hepatitis $\mathrm{C}$ infection), deep fungal infection, were 
rarely reported with $\mathrm{PG} .{ }^{1,2}$ Through a review of the literature, the association of tuberculosis with PG is extremely rare. Before now, there were only 2 reports in the literature of testicular and colonic tuberculosis accompanying pyoderma gangrenosum. ${ }^{3,4}$ Another case concerned a reactivation of tuberculosis during $\mathrm{PG}$ treatment. ${ }^{5}$ With regard to the central necrotic lymph nodes detected on the CT scan, the differential diagnosis is Wegener's granulomatosis as well as septic emboli, sarcoidosis, tuberculosis, and metastatic malignant. However, lymh nodes biopsy showed a histpathological features consistent with tuberculosis. Little is known about the possible etiologic link between infection such tuberculosis and PG. Our case further strengthens the association observed between these 2 conditions.

At present, no established protocol exists for the treatment of PG. ${ }^{6}$ The goal of therapy is directed towards the associated systemic disorder, if present. Our patient improved with antituberculous therapy.

In patients with pyoderma gangrenosum with the presence of multiple central necrotic lymph nodes, a tuberculosis manifestation of the underlying disease should be considered.

\section{References}

1. Miller J, Yentzer BA, Clark A, et al. Pyoderma gangrenosum: A review and update on new therapies. J Am Acad Dermatol 2010;62:646-54.

2. Wollina U. Pyoderma gangrenosum-a review. Orphanet J Rare Dis 2007;2:19.
3 Anton Botella F, Pena Somovilla JL, Salcedo Aguilar J, et al. Pyoderma gangrenosum associated with testicular tuberculosis. An Med Interna 1989;6:54954.

4. Kim NY, Choi JY, Lee KH, Shin JW. Pyoderma gangrenosum in a patient with colonic tuberculosis. Am J Gastroenterol 1994;89: 1257-9.

5. Matsui M, Ohtoshi E, Yamaoka J, et al. Cutaneous tuberculosis and pyoderma gangrenosum. Int J Dermatol 2000;39:3840.

6. Reichrath J, Bens G, Bonowitz A, Tilgen W. Treatment recommendations for pyoderma gangrenosum: an evidence-based review of the literature based on more than 350 patients. J Am Acad Dermatol 2005;53:27383. 